\title{
The New Phase of ISoP
}

\author{
Sten Olsson $^{1}$ (1) - Ian C. K. Wong ${ }^{1} \cdot J^{\text {Jean-Christophe Delumeau }}{ }^{1}$. \\ Mira Harrison-Woolrych ${ }^{1}$
}

Published online: 22 May 2017

(C) Springer International Publishing Switzerland 2017

\section{Introduction}

The International Society of Pharmacovigilance (ISoP) underwent transformation last year when members adopted new statutes and elected a new leadership. Reform of ISoP was initiated by the previous Executive Committee (EC) and later ratified at the general assembly with the objective of creating a more effective organization able to adapt to global changes in the science and practice of pharmacovigilance and to act in a more agile and responsive manner.

$\mathrm{We}$, the four members of the new ISoP EC, feel empowered by the mandate given to us and spurred on by the expectations on us to lead ISoP to meet the needs of the expanding pharmacovigilance profession. We have been elected to lead the society for a 3-year period in close collaboration with six additional members of the advisory board (Table 1).

ISoP began as the European Society of Pharmacovigilance (ESoP) in the early 1990s and today is a truly international professional organisation, academically oriented and embracing and driving the expansion of pharmacovigilance both geographically and professionally. Maintaining its focus on the best interests of patients exposed to pharmaceutical interventions in all healthcare systems, ISoP provides a forum for scientific

Sten Olsson

stenolssonpv@gmail.com;

http://www.isoponline.org

1 Executive Committee of the International Society of Pharmacovigilance, c/o ISoP Secretariat, 140 Emmanuel Road, London SW12 0HS, UK discussion and professional development regarding all aspects related to the safety and proper use of medicines, pharmaceutical devices and related products. ISoP is an independent not-for-profit organization funded by fees from membership and training courses and other professional events.

\section{The Diversity of the ISoP Target Audience}

National pharmacovigilance systems are now established in a clear majority of countries in the world. The level of development of these systems, as well as their cultural and societal contexts, differ widely, as does the support needed by the professionals engaged in them. The range of medicinal products covered by pharmacovigilance is no longer restricted to simple chemical molecules and herbal preparations but also extends to complex biotechnologically produced proteins that may modify immune systems and genomes as well as devices and other products containing active constituents.

The diversification and specialization of professionals who routinely consider patient safety aspects of products they are developing or monitoring has increased dramatically. Contrary to the prevailing situation 20 years ago, all categories of healthcare professionals, and patients themselves, are now expected to be alert and to report any suspicion of harm related to exposure to medicinal products, thus being part of local and global pharmacovigilance systems. The introduction of regulatory frameworks to direct and guide healthcare professionals, marketing authorization holders and scientists has led to the development of a data management and analysis industry in 
Table 1 International Society of Pharmacovigilance Advisory Board 2016-2019

\begin{tabular}{ll}
\hline Name & Position \\
\hline Executive Committee & \\
Sten Olsson & President \\
Ian C. K. Wong & Vice-President \\
Mira Harrison-Woolrych & Secretary \\
Jean-Christophe Delumeau & Treasurer \\
Board members & \\
Hilda Ampadu & Coordinator ETP Africa \\
Brian Edwards & Coordinator Chapters \\
Deirdre McCarthy & Coordinator SIGs \\
Jan Petráček & Coordinator ETP America \\
Phil Tregunno & Coordinator ETP Asia \\
Marco Tuccori & Coordinator ETP Europe \\
Past President: Hervé Le Louet & \\
\hline
\end{tabular}

ETP Education and Training Programmes

pharmacovigilance with several thousand employees globally. The introduction of innovative medicines and vaccines to combat communicable diseases, particularly in low- and middle-income countries, has necessitated public health programmes and their donor organizations to invest in and establish structures for safety surveillance of those products.

ISoP also recognizes the importance of appropriate and skilful risk communication. Study of the mechanisms involved in transferring collected safety data and information into knowledge and wisdom has led us to understand the critical role played by communication specialists, media, and medicine users themselves, and their organizations.

\section{Our Strategy and Vision for ISoP}

ISoP is committed to offering a forum for collegial discussion, exchange of information and knowledge, and the development of research methods and professional best practices for all pharmacovigilance professionals. It is obviously a challenge for ISoP to keep abreast of the needs and expectations of all its present and potential members globally, but this is certainly our ambition. With the following strategic priorities, the current EC intends to develop and expand the society in a direction that will be valued by present members, be appealing enough to attract new members and help ensure members remain in our society for many years.

\subsection{The Annual Scientific Meeting is the Most Important Event in the Life of ISoP}

Each year, the EC asks the Scientific Organizing Committee to design a programme that includes sessions of interest to members from all parts of the world and from all disciplines in pharmacovigilance with the aim of maintaining the highest scientific standards of invited lectures and accepted oral and poster presentations by delegates. Efforts will be made to coordinate the venue and the time of annual meetings when possible with the annual meetings of the World Health Organization (WHO) Programme for International Drug Monitoring to facilitate the arrangement of joint sessions and maximize the number of delegates.

\subsection{Support the Establishment and Development of National and Regional Chapters}

At present, 11 chapters have been established; these are coordinated by an assigned member of the ISoP Advisory Board (Table 2). These chapters adapt their activities to cater for the specific service and support needs identified in different geographic areas. Chapters can absorb the engagement of many ISoP members in a way that the global ISoP management cannot. Through our regional chapters, more ISoP activities can be offered, which contributes to a dynamic development of the society. For the future, the EC and the chapter coordinator will develop better support and guidance for the effective management and coordination of its chapters.

\subsection{Support the Establishment and Development of Special Interest Groups}

Similar to the ISoP chapters, the special interest groups (SIGs) attract pharmacovigilance experts in a particular discipline to exchange information and knowledge and potentially for research collaboration. ISoP currently has three SIGs, and a fourth on safety surveillance of herbal and traditional medicines is being established (Table 3). The Advisory Board also has an assigned coordinator for the SIGs.

\subsection{Promote the Benefits of ISoP Membership}

The new EC would like to better promote the advantages of being an ISoP member for existing members and to encourage recruitment and retention of members in the society. Access to the electronic version of the ISoP official journal, Drug Safety, which is included in the ISoP mem- 
Table 2 International Society of Pharmacovigilance Chapters and their coordinators in 2017

\begin{tabular}{ll}
\hline Chapter & Coordinator(s) \\
\hline Africa & Rachida Soulaymani-Benchekh \\
China & Zhou Lynn, Tang Xue, Zhang Li \\
Israel & Irene Fermont \\
Italy & Marco Tuccori \\
Latin America & Luis Alesso and Raquel Herrera Comoglio \\
Mexico & Everardo Vazquez \\
Middle East & Mayada Alkhakany and Thamer Alshammary \\
North America & Under negotiation \\
South East Europe & Katarina Ilic \\
Switzerland-Austria & Stefan Russmann and Victoria Rollason \\
Western Pacific region & Richard Hill \\
\hline
\end{tabular}

Table 3 International Society of Pharmacovigilance special interest groups and their coordinators in 2017

\begin{tabular}{ll}
\hline SIG & Coordinators \\
\hline Women's medicines & Mira Harrison-Woolrych \\
Risk communication & Priya Bahri \\
Risk minimization methods for Asia countries & Jean-Christophe Delumeau \\
Herbal and traditional medicines & Joanne Barnes \\
\hline
\end{tabular}

$S I G$ special interest group bership fee, needs to be better promoted and explained. ISoP may involve Drug Safety in training webinars on how to write good scientific articles-access to such webinars would be an additional membership bonus.

\subsection{Improve Opportunities for Active Dialogue Between ISoP Management and Members}

We intend to improve opportunities that will allow members to regularly make suggestions and express their preferences for ISoP activities. A recent step in this direction is the opening of an ISoP account on Twitter (@ISoPonline) in addition to the established ISoP group on LinkedIn. Further presence in social media is being planned. The ambition is to make the email addresses of ISoP chapter and SIG coordinators publicly available to stimulate active membership dialogues.

\subsection{Support Education and Training in Pharmacovigilance and Risk Minimization Strategies}

Significant work carried out by previous ISoP EC members and others has resulted in a structured framework for competence development in pharmacovigilance through the WHO-ISoP Core Modular Curriculum [1]. ISoP intends to continue collaborating with partners to create tools and materials for education delivery, building on recent suggestions of a crowdsourcing approach [2].

\subsection{Coordinate ISoP Global Activities} in Pharmacovigilance with Those of the World Health Organization and the Council for International Organizations of Medical Sciences

In early 2017, ISoP and managers of pharmacovigilance activities by the WHO and the Council for International Organizations of Medical Sciences (CIOMS) met with the intention of establishing good communication and coordination of activities. The WHO shared information about its Coalition of Interested Parties (CIP) initiative and invited ISoP to be a part of its strategic advisory group for the CIP in pharmacovigilance. Both the WHO and the CIOMS invited ISoP to collaborate in identifying domain experts among its members for working groups and training activities carried out by them. Several opportunities for joint conferences were identified.

\subsection{Continue and Expand the Training Collaboration with the Uppsala Monitoring Centre}

The successful training courses carried out jointly with the Uppsala Monitoring Centre (UMC) since 2014 will continue and be enhanced. Based on a renewed memorandum of understanding, UMC support is now extended from one course to two courses per annum [3]. For 2017, courses are being planned in Latin America and in China in collaboration with the new ISoP Chinese chapter. 


\subsection{Minimize Administrative Obstacles for Potential Members Around the Globe to Join the Society}

The present system for individual membership and internet-based payment of fees is convenient for some members but presents administrative problems for others who would like to join the society. ISoP intends to review the routines currently in use so that everyone wanting to join the ISoP pharmacovigilance community will feel welcome and will be assisted in finding a suitable membership model and an easy way of transferring funds.

\section{The Way Forward for ISoP as a Global Pharmacovigilance Forum}

We, the EC of ISoP, think our society has a unique position as a global independent scientific and professional organization, attracting pharmacovigilance scientists and professionals from all relevant sectors in society across all geographic regions. ISoP is an inclusive and independent society that operates on not-for-profit principles. Members describe our society as 'friendly' and 'a pharmacovigilance family', and we wish to promote and encourage this environment for professional networking. With the new statutes and by-laws, we are equipped with an organizational framework that gives sufficient flexibility for us to adapt to the needs of the different stakeholders involved in pharmacovigilance. We can demonstrate a local and regional presence coordinated by a global management structure that is well aligned with other global actors in the pharmacovigilance discipline. We invite current and prospective members to engage in dialogue with us with the aim of continuously developing our services and practices and allowing us to remain a society with high scientific standards that focusses on the well-being of patients exposed to medicinal products.

\section{Compliance with Ethical Standards}

Funding No sources of funding were used to assist in the preparation of this article.

Conflicts of interest Sten Olsson, Ian C.K. Wong, Jean-Christophe Delumeau and Mira Harrison-Woolrych have no conflicts of interest that are directly relevant to the content of this article.

\section{References}

1. Beckmann J, Hagemann U, Bahri P, et al. Teaching pharmacovigilance: the WHO-ISoP core elements of a comprehensive modular curriculum. Drug Saf. 2014;37(10):743-59.

2. Bate A, Beckmann J, Dodoo A, et al. Developing a crowdsourcing approach and tool for pharmacovigilance education material delivery. Drug Saf. 2017;40:191-9.

3. International Society of Pharmacovigilance. Training: ISoP-UMC training. http://isoponline.org/training/isop-umc-training-courses/. Accessed 17 May 2017. 